\title{
Hipótesis de progresión del conocimiento biológico y del conocimiento didáctico del contenido biológico. Parte I: referentes teóricos
}

\author{
Édgar Orlay Valbuena Ussa
}

Artículo recibido: 07-07-2011 y aprobado: 15-07-2011

Progression hypothesis of biological knowledge and pedagogical knowledge of biological content. First part: theoretical backgrounds

Resumen: Este artículo, constituye el primero de una serie de dos, consistentes en el abordaje de dos constituyentes fundamentales del conocimiento profesional del profesor de Biología. Primero se presenta la perspectiva del autor sobre el conocimiento profesional del profesor de Biología, y el conocimiento didáctico del contenido biológico (CDCB); luego, a partir de la revisión de antecedentes y de referentes teóricos, se propone una hipótesis de progresión tanto para el conocimiento biológico (estructuras sintáctica y sustantiva) como para el CDCB. Para el caso del primer conocimiento, la hipótesis plantea dos extremos: la perspectiva fisicalista y la visión estructural-sistémica; en cuanto al CDCB, la hipótesis plantea dos polos: el reduccionismo biológico y la perspectiva integradora-transformadora. Posteriormente, en el segundo artículo se presentarán los resultados de investigación, a partir del análisis de datos de estudios obtenidos con futuros profesores de Biología.

Palabras clave: Conocimiento Biológico, Conocimiento Didáctico del Contenido Biológico, Hipótesis de Progresión, Formación docente.
Abstract: This article is the first of a series of two, consisting of two fundamental constituents addressing professional knowledge of biology teachers. First, it presents the perspective of the author on the professional knowledge of biology teacher and the pedagogical knowledge of biological content. Then, the progression hypothesis of biological knowledge and pedagogical knowledge of biological content is proposed based on the background and related theoretical review. Regarding the first knowledge the hypothesis states two extremes: the physicalist perspective and the structural- systematic vision; with respect to the pedagogical knowledge of biological content, the hypothesis states two poles: biological reductionism and transformative- integrative perspective. Subsequently, in the second article, the author will present the research project results obtained from the data analysis from studies that were carried with pre service biology teachers.

Key words: Biological knowledge, pedagogical knowledge of biological content, progression hypothesis, teachers' education.

Profesor Universidad Pedagógica Nacional. Coordinador Grupo de Investigación Conocimiento Profesional del Profesor de Ciencias. valbuena@pedagogica.edu.co, edgarorlay@hotmail.com 


\section{Introducción}

El profesor de ciencias y el de Biología en particular cuenta con un conocimiento específico, que lo diferencia de otros profesionales y lo faculta para enseñar esta disciplina, la cual a su vez se identifica por contar con una epistemología diferente a la de otras ciencias. Sin embargo, frente a la anterior premisa, la caracterización de dicho conocimiento profesional es apenas emergente, haciéndose necesario realizar investigaciones para visibilizarlo, esto, a partir de la sistematización y análisis de estudios realizados por la comunidad de académicos del campo de la enseñanza de las ciencias, y tomando como base el pensamiento, las concepciones y las prácticas pedagógicas de profesores. Esto, con miras a identificar los componentes, las fuentes, la estructura y la construcción del conocimiento profesional del profesor de Biología, lo cual tiene relevantes implicaciones, entre otras en: la formación de profesores, el diseño y evaluación de textos escolares de enseñanza de la Biología, la definición de políticas sobre enseñanza de la Biología, la caracterización y mejoramiento de las prácticas pedagógicas de profesores de Biología, y la caracterización y formulación de estrategias de enseñanza de la Biología (Valbuena 2007, 2009a).

Una de las prioridades del grupo de investigación Conocimiento Profesional del Profesor de Ciencias, ha sido la caracterización del conocimiento profesional del profesor de Biología, haciendo énfasis en el conocimiento biológico y el conocimiento didáctico del contenido biológico (Valbuena, 2007; Bernal, 2007; Gutiérrez, A., 2007; Sánchez, 2007; Puentes, 2008; Amórtegui y Correa, 2009; Morales, 2010; Valbuena et al 2010a; Valbuena et al 2010b; Amórtegui, 2011). Para ello, hemos adoptado la perspectiva epistemológica y metodológica propuesta por el grupo Didáctica e Investigación en la Escuela -DIE-, correspondiente a la hipótesis de progresión (García, 1998; Porlán y Rivero, 1998), según la cual, el conocimiento no es único y está constituido por diferentes niveles tentativos de progresiva complejidad.

La hipótesis de progresión se concreta en la formulación de diferentes niveles de complejidad, desde unos más simples, o de partida (que generalmente se corresponden con el conocimiento mayoritario entre los profesores), hasta unos más complejos que corresponden con el conocimiento deseable. La hipótesis de progresión se entiende así, como el marco de referencia para el formador. Todo ello, no implica que los futuros profesores tengan que recorrer y superar un itinerario lineal para alcanzar el conocimiento deseable. Esta perspectiva permite además: detectar dificultades y obstáculos en los estudiantes; evidenciar la diversidad de concepciones; asumir la formación de profesores y el conocimiento profesional como un proceso dinámico y cambiante. Así pues, la hipótesis de progresión, amplía el panorama de investigación, permitiendo caracterizar el conocimiento del profesor de tal forma que se da cabida a una gama de conocimientos, lo cual favorece los procesos formativos en tanto facilita la interpretación de la realidad educativa y la intervención en los procesos de formación docente. Así, por ejemplo, la indagación de concepciones de los docentes, bajo esta perspectiva, da la posibilidad de estructurarlas en un gradiente que favorece la interpretación de dichas concepciones, así como el 
trabajo didáctico con las mismas, con miras a su transformación y enriquecimiento. En este sentido, la hipótesis de progresión no constituye "un itinerario ineludible por el que deban pasar todos los profesores siguiendo una trayectoria lineal, progresiva y ascendiente, sino que debe entenderse de manera flexible, admitiendo los ciclos y fluctuaciones que inevitablemente se dan en los procesos formativos" (Porlán y Rivero, 1998: 147).

En esta dirección, el presente artículo, pretende aportar elementos conceptuales para la formulación de la hipótesis de progresión de dos categorías del conocimiento del profesor de Biología. Para tal fin, inicialmente se presenta la propuesta del autor respecto a la estructuración de dicho conocimiento, posteriormente se resume la sistematización realizada con base en la revisión de investigaciones sobre el conocimiento del profesor de Biología y finalmente se presenta la propuesta de hipótesis de progresión sobre el conocimiento biológico, y el conocimiento didáctico del contenido biológico, tomando como base fundamentalmente los referentes teóricos. Cabe advertir, que en un próximo artículo se presentarán resultados del enriquecimiento de estas hipótesis, atendiendo a los hallazgos de investigación con futuros profesores de Biología.

\section{Metodología}

Este estudio corresponde a la perspectiva de investigación cualitativa, concretamente se realizó desde la perspectiva interpretativa. Corresponde a un estudio documental a partir de la revisión bibliográfica en fuentes especializadas en el campo de la epistemología de la Biología y la didáctica de las ciencias. Para tal fin se desarrollaron las siguientes fases:
- Identificación de fuentes de información especializadas (libros de epistemología de la Biología y didáctica de las ciencias, así como revistas de enseñanza de las ciencias y de formación de profesores).

- Revisión de los documentos y sistematización, teniendo como referente el conocimiento del profesor. En el caso de la revisión de antecedentes sobre el conocimiento del profesor de Biología, para la sistematización se tuvieron en cuenta los siguientes elementos: población de estudio, enfoque metodológico (incluyendo los instrumentos empleados para la obtención de datos y los métodos para el procesamiento de los mismos), los componentes del conocimiento del profesor abordados, y los principales resultados.

- Formulación de propuestas conceptuales sobre el conocimiento profesional del profesor de Biología y de las hipótesis de progresión sobre el conocimiento biológico y el conocimiento didáctico del contenido biológico. Esto, fundamentalmente a partir de la contrastación y análisis de los referentes teóricos y de los antecedentes de investigación.

\section{Resultados y discusión}

Los resultados se presentan en cuatro grandes apartados. Así: el posicionamiento teórico frente al conocimiento profesional del profesor de Biología, haciendo especial énfasis en el conocimiento didáctico del contenido biológico (se describe la propuesta del autor al respecto); las tendencias en estudios sobre conocimiento del profesor de Biología; la hipótesis de progresión sobre 
el conocimiento biológico y la hipótesis de progresión sobre el conocimiento didáctico del contenido biológico.

\section{El Conocimiento Profesional del Profesor y el Conocimiento Didác- tico del Contenido Biológico como marcos de referencia}

El conocimiento del profesor ha sido objeto de investigaciones desde diferentes perspectivas en las últimas décadas. $\mathrm{Al}$ respecto, Fenstermacher (1994) pone de manifiesto que los estudios en las décadas de los 60 y 70 no hacían referencia de manera explícita al conocimiento del profesor y el énfasis estaba puesto en caracterizaciones de la efectividad del profesor (perspectiva de procesoproducto). En contraste, a partir de la década del 80 se desarrollan investigaciones asumiendo a los profesores como sujetos de saber, centrando los esfuerzos en estudiar el conocimiento práctico del profesor. Así, para dicho autor en las primeras investigaciones predominaba el conocimiento del profesor como componente estático; mientras que en la segunda se asumía dicho conocimiento como componente dinámico.

La perspectiva epistemológica con la que se identifica el presente artículo corresponde al conocimiento del profesor como un conocimiento práctico profesional. Es decir, un conocimiento que deviene de la práctica pedagógica y didáctica del profesor, a partir de variadas fuentes (experienciales, afectivas, teóricas y culturales) y que posee una estructuración compleja y única, compuesta por diferentes constituyentes (conocimiento de la materia que se enseña, conocimiento del contexto, conocimiento pedagógico, conocimiento metadisciplinar y conocimiento didáctico del contenido); un conocimiento de carácter profesional en tanto le permite al profesor identificar, reflexionar, sistematizar, autorregular y transformar el proceso de enseñanza del cual él es protagonista.

Como se puede interpretar, de la rigurosa revisión realizada por Abell (2007), pese al gran volumen de investigaciones sobre el conocimiento del profesor, aún no existe una "madurez" teórica y metodológica al respecto. Así, por ejemplo, existe una diversidad de propuestas sobre la estructuración de dicho conocimiento; además, se denota una dispersión terminológica para referirse de una manera un tanto indistinta al mismo: pensamiento, concepciones, creencias, valores, saberes y conocimientos.

A partir de la revisión y análisis de diferentes propuestas sobre la estructura del conocimiento del profesor (Shulman, 1986 a,b, 1987; Hashweh, 1985; Grossman, 1990; Marcelo, 1987; Porlán y Rivero, 1998; Carlsen, 1999, Gess-Newsome y Lederman, 1999; Magnusson, Krajcik y Borko, 1999; MorineDershimer y Kent, 1999; Tardif, 2004; Abell, 2007, 2008; Park y Oliver, 2008), y teniendo en cuenta resultados propios de investigación (Valbuena, Castro y Sierra, 2006; Valbuena, 2007; Castro y Valbuena, 2007; Castro, et al, 2009; Morales y Valbuena, 2009; Valbuena 2009a; Valbuena, 2009b; Valbuena et al, 2010b), el autor de este artículo se identifica con la siguiente propuesta de estructuración. Así, considera que los principales componentes del conocimiento profesional del profesor de Biología son (ver Figura 1): 
- El conocimiento y las concepciones de la materia que se enseña (Subject Matter Specific), en este caso: el conocimiento biológico.

- El conocimiento y las concepciones pedagógicas y didácticas.

- El conocimiento del contexto.

- El conocimiento y las concepciones metadisciplinares.

- El conocimiento cultural.

- El conocimiento didáctico del contenido específico. En este caso: el conocimiento didáctico del contenido biológico.

En la figura antes citada, se representan las relaciones entre los componentes, haciendo referencia concretamente al conocimiento del profesor de Biología. Se enfatiza en la relevancia del conocimiento didáctico del contenido biológico -CDCB- como elemento integrador en la constitución del conocimiento profesional del profesor de Biología. Como se puede observar, en la parte superior del esquema (dentro del marco) se encuentran los conocimientos de tipo teórico, es decir los académicos, los cuales se adquieren durante la educación formal y tradicionalmente son los que predominan en los programas de formación del profesorado.

En forma de marco se representan los conocimientos contextuales, cultural y el metadisciplinar. El ubicar los conocimientos contextuales en el marco del diagrama, se identifica con el enfoque de conocimiento del profesor propuesto por Carlsen (1999), y obedece a que todos los conocimientos y acciones del profesor están influenciados por las condiciones y características del entorno, desde las políticas internacionales y nacionales sobre la educación, hasta las particularidades de los alumnos y el aula de clase en que se ejerza la profesión docente.

No se puede desconocer que el conocimiento del profesor está influenciado por la cultura. Esto, teniendo en cuenta que el maestro no se puede despojar de las representaciones, concepciones y saberes que devienen de la cultura en la cual se han formado él y sus estudiantes. Al respecto, existe toda una línea de investigación que problematiza las relaciones entre los saberes y conocimientos de índole científico, respecto a los construidos en las diferentes culturas. Ello corresponde a desarrollos importantes desde la perspectiva de la interculturalidad (entre otros: Cobern y Loving, 2001, Aikenhead, 2007; Lee y Luykx, 2007; Oliver, 2007). Por ejemplo, estudios como el de El-Hani y Sepúlveda (2006) ilustran, para el caso de la enseñanza y el aprendizaje de conceptos biológicos como la evolución, la importancia de no desconocer otras explicaciones al respecto, diferentes a la científica, como es el caso de las aportadas por las religiones.

De igual manera, el conocimiento metadisciplinar se representa formando parte del marco, esto en consonancia con los planteamientos del Proyecto Curricular Investigación y Renovación Escolar (IRES), en el sentido de que este conocimiento constituye el marco de referencia que posibilita la estructuración de los sistemas de ideas de los alumnos, necesario para la construcción del conocimiento escolar, que orienta el establecimiento de interrelaciones, conlleva al acercamiento entre conocimientos parciales (García, 1998) y actúa 
como organizador del conocimiento profesional (Porlán y Rivero, 1998).

En la base de la figura 1 (dentro del marco), a manera de soporte, se representan los conocimientos experienciales y las concepciones personales del profesor de Biología. Las experiencias de los profesores, tal y como lo resalta Tardif (2004), constituyen saberes fundamentales en la estructuración de su desarrollo profesional.

Así mismo, la epistemología personal de los docentes sobre: el conocimiento en general, la enseñanza y el aprendizaje, la Ciencia y la disciplina específica que se enseña, constituye un referente clave en el conocimiento profesional. La ubicación de este tipo de conocimiento en la base del esquema, obedece a que tanto los saberes experienciales como las concepciones del profesor son de carácter implícito, arraigado y funcional, lo cual conlleva a que sean los más próximos a las conductas del profesor. Tal y como lo afirman Barnett y Hodson (2001) las creencias, valores y experiencias personales del docente, orientan en buena medida la manera como se interpretan e implementan los currículos.

En la parte central de la figura, en medio de los conocimientos teóricos, culturales, prácticos y contextuales, se ubica el conocimiento didáctico del contenido biológico -CDCB-. De manera similar a los enfoques de Grossman (1990), Carlsen (1999), Magnusson, Krajcik y Borko (1999), Morine-Dershimer y Kent (1999), y transformador de GessNewsome (1999a), quienes le asignan un lugar preponderante al Pedagogical Content Kcnowledge -PCK- (que en la presente propuesta se considera al CDC) el elemento nuclear alrededor del cual se procesan, transforman, complejizan e integran los demás constituyentes del Conocimiento Profesional docente. $\mathrm{Al}$ respecto, cabe señalar que se utiliza el término CDC y no PCK, teniendo en cuenta las implicaciones del conocimiento didáctico para la enseñanza de un contenido específico, y no obedece a una simple traducción. Dadas las limitaciones de espacio, en este artículo no se desarrolla esta precisión.

Se entiende el CDCB como aquel conocimiento que le hace posible al profesor enseñar Biología, lo cual trasciende una mera adaptación o simplificación del conocimiento biológico (el de origen científico) al contexto y realidades escolares. Se trata de utilizar un conocimiento profesional para lograr lo que García (1998) denomina la integración y transformación didáctica, para hacer posible la construcción de un conocimiento escolar (en este caso sobre la Biología) el cual posee un estatus epistemológico diferenciado. Dicho CDCB le permite al profesor responder a preguntas tales como: ¿qué contenidos de Biología enseñar?, ¿se trata solamente de enseñar contenidos biológicos?, ¿además de los datos y conceptos biológicos qué procedimientos y actitudes enseñar?, ¿cómo enseñar dichos contenidos?, ¿cómo conocer y utilizar didácticamente las ideas de los alumnos acerca de contenidos biológicos que se enseñan?, ¿cómo estructurar los contenidos de enseñanza?, ¿lo importante es enseñar gran cantidad contenidos, o se trata de estructurarlos y organizarlos para su enseñanza?, ¿cómo abordar las concepciones, representaciones, saberes y conocimientos que los estudiantes tienen, los cuales derivan de contextos culturales específicos?, ¿cómo 
indagar y qué hacer con las experiencias, necesidades, intereses y expectativas de los alumnos frente a un tema determinado?, ¿qué dificultades existen en la enseñanza-aprendizaje de la Biología, y cómo superarlas?, ¿qué propósitos tiene la enseñanza de la Biología en relación con las necesidades sociales, contextuales y personales?, ¿qué, cómo, quién, cuándo, y para qué, evaluar los aprendizajes de los contenidos biológicos enseñados?

Como se puede apreciar en el esquema se representan flechas en doble sentido: desde el CDCB hacia los componentes teóricos, experienciales y concepciones, y viceversa. Con ello se quiere indicar que para la constitución del CDCB confluyen diferentes conocimientos, y a la vez, que el CDCB constituye un elemento que hace posible la reflexión de las fuentes. Esto, posibilita la construcción del Conocimiento Profesional a partir de la reflexión. De otra parte, los componentes del CDCB se representan en recuadros con líneas interrumpidas y relacionando cada uno de ellos con el Conocimiento Escolar Biológico. Ello, con el fin de destacar las interrelaciones entre los componentes, lo cual se hace más posible, en la medida que los maestros desarrollen capacidades metacognitivas que les permita, además de hacer explícitos los componentes, promover su integración.

A continuación, se hace referencia a otro componente fundamental del conocimiento profesional del profesor de Biología: el conocimiento de la disciplina que se enseña (en este caso: el conocimiento biológico), originalmente denominado en el ámbito anglosajón Subject Matter Specific. Éste comprende los contenidos concretos de enseñanza, y la forma como están organizados en su estructura sustantiva y sintáctica (según la clasificación de Schwab, 1978, citado en Shulman, 1986b). La estructura sustantiva hace referencia a "la variedad de formas como los conceptos y principios básicos son organizados para incorporar los hechos" (Shulman, 1986b: 9). Como lo señala Marcelo (1999),el conocimiento sustantivo no se limita a contemplar conceptos y principios generales de la disciplina sino que incluye "el conocimiento de los marcos teóricos, tendencias y la estructura interna de la disciplina en cuestión" (p. 156). La estructura sintáctica se refiere a las maneras como se verifican o falsean, se validan o invalidan los contenidos, "la sintaxis de la disciplina aporta las reglas para determinar qué aseveración tiene mayor nivel de justificación" (Shulman, 1986b: 9). Grossman manifiesta que la estructura sintáctica incluye la sustantiva y tiene que ver con el dominio del docente de "los criterios aceptados, y que se utilizan por los miembros de la comunidad científica para orientar la investigación en dicho campo. Son los medios por los cuales se introduce y acepta por la comunidad un nuevo conocimiento" (1990: 29).

Según Gess-Newsome (1999b) y Abell (2007), el conocimiento disciplinar específico es necesario (incluyendo su estructura y naturaleza) ya que le posibilita al profesor: establecer relaciones entre los conceptos que se enseñan; identificar los principios fundamentales de la disciplina, y en esa medida seleccionar, secuenciar y transformar los contenidos de enseñanza prioritarios; identificar las aplicaciones que puedan 
Hipótesis de progresión del conocimiento biológico y del conocimiento didáctico del contenido biológico. Parte I: referentes teóricos

tener los contenidos, a la cotidianidad de los alumnos; realizar síntesis de contenidos; diseñar, implementar y/o adaptar prácticas de laboratorio; formular preguntas y problemas con niveles de complejidad acordes con los propósitos de la enseñanza; abordar y resolver problemas; detectar las ideas erróneas de los alumnos; y evaluar y seleccionar textos escolares, teniendo en cuenta la veracidad y la estructura organizativa de los contenidos.

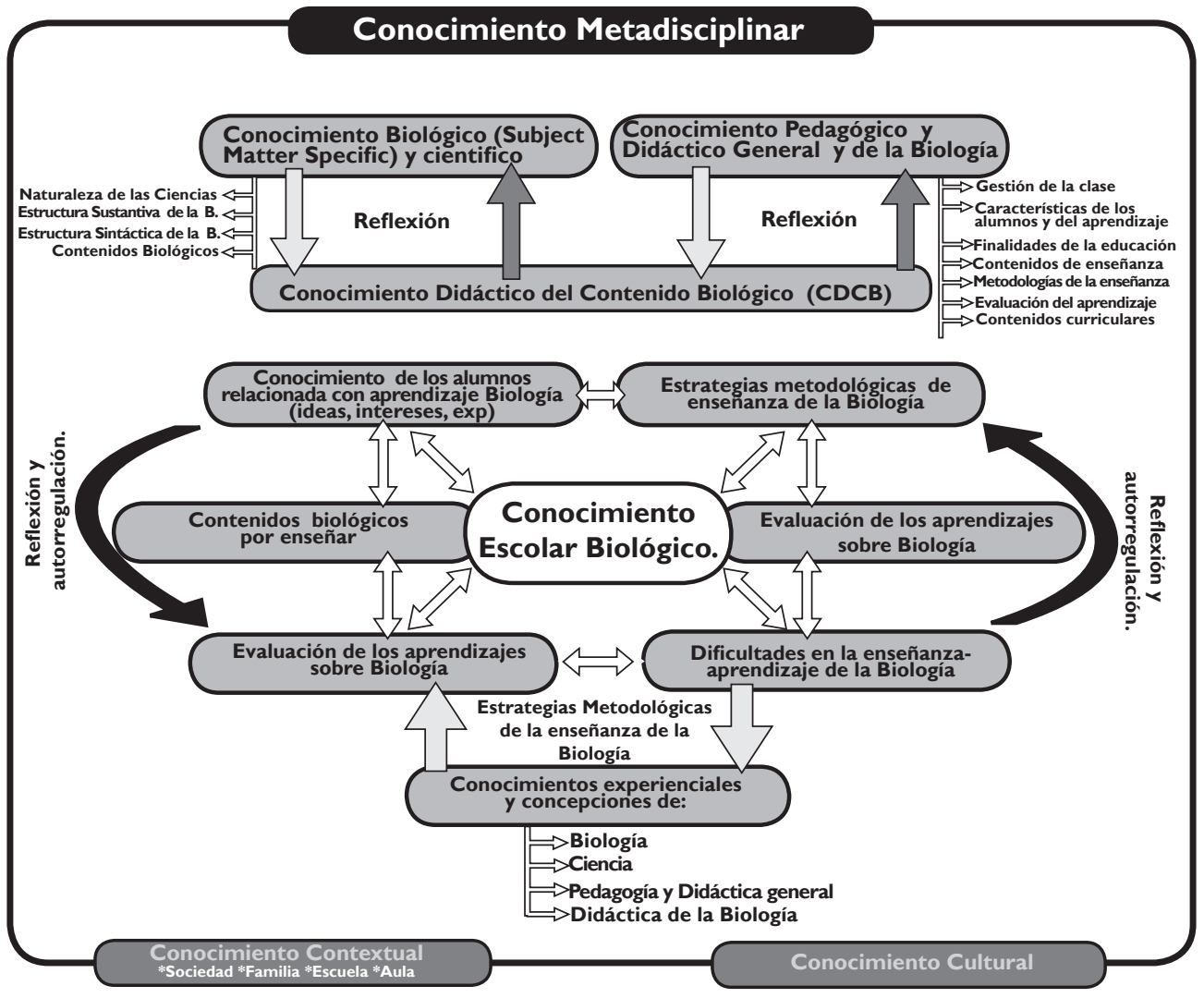

Figura 1. El Conocimiento Didáctico del Contenido Biológico como núcleo integrador en la construcción del Cocimiento Profesional del Profesor de Biología. T.I.D.: Transformación e Integración Didáctica. (Actualizado a partir de Valbuena, 2007). 


\section{Investigaciones sobre el conocimiento del profesor de Biología}

De la revisión realizada, se deduce que la mayoría de investigaciones sobre el conocimiento del profesor de Biología, hace referencia al conocimiento biológico (Subject Matter), específicamente a la estructura sustantiva. Un estudio realizado por Baxter, Richert y Saylor (1985, citado en Gess-Newsome, 1999a:65) con cinco profesores noveles quienes organizaron diez temas considerados centrales en la Biología, encontró diferencias en la identificación de tópicos estructurantes y en la complejidad en las relaciones establecidas entre los conceptos. Hoz, Tomer, y Tamir (1990) analizaron mapas conceptuales elaborados por profesores de Biología, a partir de listados (célula, virus, ADN, energía, enzima, respiración, fotosíntesis, hongo y metabolismo, ATP, degradación de grasas, glucosa, síntesis de proteínas, almacenamiento de carbohidratos, difusión, mitosis, ribosoma, citoplasma, pp: 976), encontrando que independientemente de la experiencia docente, los profesores tienen deficiencias en su conocimiento biológico.

Hauslein, Good y Cummins (1992), investigaron la manera como cuatro grupos de profesores de Biología (treinta y dos) con diferente experiencia en la enseñanza, y un grupo de biólogos (siete), organizaban 37 tópicos de Biología (sucesión de especies, selección natural, evolución, variación de organismos, adaptación ambiental, competencia entre organismos, migración, fertilización, ovulación, reproducción, menstruación, apareamiento, herencia, coagulación sanguínea, circulación sanguínea, acción refleja, excreción, digestión, regulación de fluidos sanguíneos, difusión, ósmosis, transporte activo, transporte celular, reacción enzimática, mitosis, ruptura celular, meiosis, regeneración de tejido, diferenciación celular, crecimiento, simbiosis, parasitismo, fotosíntesis, ciclo del carbono, polinización, metabolismo, respiración, pp: 943), encontrando diferencias en la estructuración de los conceptos, siendo más elaborada (en cuanto a la complejidad de interrelaciones) en el caso de los profesores experimentados y los biólogos; todos los profesores organizaron los tópicos teniendo en cuenta la manera como se enseñan, y estructuraron los contenidos a manera de contenidos para exámenes; los biólogos mostraron estructuras del conocimiento biológico que les permitía diferenciar las causas próximas (como las propias de los procesos fisiológicos) de las remotas de la Biología (por ejemplo, las relacionadas con la evolución), resultado coherente con los planteamientos de Mayr (1998, 2006), que ponen de manifiesto particularidades sustantivas del conocimiento biológico.

En la investigación realizada por Gess-Newsome y Lederman (1993), con diez futuros profesores de Biología (en el contexto de tres cursos: métodos y estrategias de ciencias de Secundaria, microenseñanza y prácticum) se encontró que los docentes en formación admitían que nunca antes se habían preguntado sobre la estructura de los contenidos, y reconocían la dificultad para su organización, atribuyéndole esa limitación a la manera como abordaban este aspecto a lo largo de su proceso formativo como estudiantes. Los esquemas realizados eran simples y se 
correspondían con los contenidos de los cursos y libros de texto universitarios. En su mayoría, se limitaban a enumerar las ramas o subdisciplinas de la Biología, sin hacer referencia a conceptos fundamentales y centrales como tales.

Estos resultados concuerdan con los obtenidos por los mismos investigadores (Lederman, Gess-Newsome y Latz, 1994) con doce futuros profesores de Ciencias de Secundaria (siete de ellos de Biología), al inicio de la fase formativa se encontró que la estructura del conocimiento biológico corresponde a un simple listado de temas, de una forma similar a los programas de cursos en la Universidad. Luego de la reflexión de los resultados de los cuestionarios y, tras el desarrollo del proceso formativo, los futuros profesores estructuraron los contenidos biológicos de una forma más integrada (por ejemplo, una de las estudiantes presentó los componentes del conocimiento biológico como parte de un paraguas, es decir como componentes de un todo) incluyendo además de los campos o subdisciplinas biológicas, conceptos fundamentales como lo vivo y el pensamiento humano, a manera de dimensiones nucleares de integración. No obstante, a pesar de replantear sus propuestas de estructuración de los tópicos, expresaron la dificultad para llevar esas propuestas de organización a la práctica en la enseñanza. De otra parte, destacaron la importancia de establecer interrelaciones de conceptos al trabajar con los alumnos, y de tener una perspectiva integradora del cconocimiento biológico para facilitar de esta manera la comprensión por parte de los alumnos. Los futuros profesores manifestaron que la estructura del cconocimiento biológico se basa principalmente en los programas de Biología estudiados tanto en la Secundaria, como en la Universidad (Gess-Newsome y Lederman, 1993; Lederman, Gess-Newsome y Latz, 1994). El mismo resultado fue encontrado en profesores de Biología con experiencia, lo cual explica el hecho de que en los esquemas predominen los listados de ramas de la Biología y que las relaciones entre los elementos de los diagramas sean escasas. Al respecto, los autores llaman la atención sobre la manera fragmentada como se enseñan los diferentes campos de la Biología en las instituciones educativas. En relación con la influencia de la experiencia docente, en el nivel de complejidad de la estructura del conocimiento biológico, Gess-Newsome y Lederman (1995), en el estudio de caso realizado con cinco profesores experimentados, encontraron que incluso profesores de Biología con experiencia entre 7 y 26 años presentan estructuras basadas en contenidos discretos que rara vez integran.

En la investigación de Lederman, Gess-Newsome y Latz (1994) se encontró que tras el desarrollo de los cursos de formación inicial, los futuros profesores evolucionaron en la estructuración del conocimiento biológico. Es así como los esquemas realizados por los estudiantesprofesores son más consistentes y muestran una mayor integración entre los componentes. Incluyen, además de los campos o subdisciplinas biológicas, conceptos fundamentales como lo vivo y el pensamiento humano, a manera de dimensiones nucleares de integración. Los futuros profesores de las investigaciones a las que se está haciendo referencia manifiestan que la 
estructura del conocimiento biológico se basa principalmente en los programas de Biología que han estudiado, tanto en la Secundaria, como en la Universidad (Gess-Newsome y Lederman, 1993; Lederman, Gess-Newsome y Latz, 1994).

Estudios como el de Hashweh (1987), relacionan el conocimiento biológico con la enseñanza. Este autor señala que el conocimiento biológico que posea el profesor, incide en la manera como éste estructura los contenidos, y las actividades de la enseñanza. De los seis docentes con experiencia estudiados por él (tres de Biología y tres de Física), tan sólo uno de Biología tiene un enfoque molecular del proceso de la fotosíntesis (en el cuestionario se refiere a $\mathrm{CO}_{2}, \mathrm{H}_{2}$, $\mathrm{O}_{2}, \mathrm{NADPH}^{+}$, y glucosa) y energético a la vez (energía lumínica, energía química de enlace, energía para biosíntesis de moléculas, energía para ruptura de moléculas). Dicho profesor, a diferencia de los otros cinco, establece relaciones entre los procesos metabólicos de la fotosíntesis y la respiración celular, desde las perspectivas de la transformación de materia y energía. De igual manera, relaciona fácilmente la fotosíntesis con conceptos ecológicos como la red trófica. En contraste, los otros profesores se limitan a describir estructuras anatómicas vegetales (por ejemplo de la hoja) y procesos ecológicos generales. Igualmente, las relaciones que muestran los mapas conceptuales de estos docentes son más simples, al incluir menos elementos y menos interconexiones. Otro aspecto que muestra el estudio de este autor, es que los profesores de Biología, al contrario de los de Física, identifican la organización de los contenidos del capítulo de energía y célula, de un libro de texto que se les había suministrado para su análisis. Cabe señalar, que dicha investigación, hace referencia al aspecto relacionado con los contenidos del Conocimiento Biológico y su influencia en la enseñanza, y no hace un análisis de otros aspectos del conocimiento disciplinar como los referentes epistemológicos.

Investigaciones como la de Veal y Kubasko (2003), muestran la incidencia del conocimiento biológico en la enseñanza, la cual se refleja en: la selección y organización tanto de contenidos, las estrategias de enseñanza, la utilización de un vocabulario rico, el establecimiento de integración entre diferentes conceptos (evolución, genética, bioquímica y clasificación de las especies). Carlsen (1993) investigando el caso de profesores inexpertos de Biología, halló que cuando se trata de temas que no le son familiares, los docentes dedican mayor tiempo, la frecuencia de preguntas a los alumnos es mayor (pero con niveles de dificultad cognitiva mínimos) y las prácticas de laboratorio son abiertas, la participación que predomina es la del profesor (fundamentalmente mediante sus explicaciones). En contraste, para la enseñanza de temas que le resultan más familiares al docente, se propician más espacios de participación de los alumnos, la revisión de tareas es más frecuente, hay una menor cantidad de preguntas a los alumnos, y una mayor participación de los estudiantes en grupos de trabajo. Por su parte, Hauslein, Good y Cummins (1992), explican que no se trata de que el conocimiento del contenido del profesor, adquiera per se, el nivel del conocimiento biológico del científico, sino que es fijado mediante los trabajos pedagógicos en la estructura particular 
del currículo, por eso se mantiene fijo y no llega a ser tan "fluido" como el del científico.

Cabe citar otras investigaciones que enfatizan en el conocimiento de los profesores sobre conceptos biológicos específicos, tales como: nutrición (Benson,1989) célula (Barass, 1984; Douvdevany, Dreyfus y Jungwirth, 1997) y respiración (Sanders, 1993).

Por otra parte, en Colombia, la investigación realizada por Chona et al. (1998) encontró que los veintitrés profesores colombianos estudiados, creen que la Biología "es una mezcla de disciplinas, está cruzada por la Física y la Química” (Chona et al., 1998: 98) y tiene implicaciones de orden político, económico y social, especialmente en lo relacionado con la Ecología, la salud, la biodiversidad y la conservación. Por su parte, Valbuena et al.(2010 b), identifican los procesos formativos que favorecen la construcción del conocimiento profesional del profesor de Biología en futuros profesores, concretamente mediante el diseño de unidades didácticas, y la formulación de anteproyectos pedagógicos relacionados con la enseñanza de la Biología, destacan la relevancia de promover en los procesos formativos, la metacognición en los docentes en formación, los cuales permiten en los futuros profesores: identificar los conocimientos y saberes que requiere un profesor para poder enseñar Biología; reflexionar sobre los diferentes componentes del conocimiento profesional del profesor de Biología, tomando como base la planificación que hacen; y replantear sus propuestas de enseñanza.

Los resultados antes presentados ponen de manifiesto la emergencia de la producción respecto al conocimiento del profesor de Biología, lo cual ratifica la necesidad de realizar este tipo de investigaciones, con miras a caracterizar los elementos epistemológicos de dicho conocimiento, así como al mejoramiento en la formación y promoción docente (políticas de formación, selección y promoción de profesores, organización de programas curriculares de formación, etc.).

\section{Hipótesis de progresión del conocimiento biológico (C.B.)}

Concretamente, hace referencia a la estructura sintáctica, las finalidades, y la estructura sustantiva del conocimiento biológico (ver Tabla 1).

A partir de la revisión bibliográfica (Blandino, 1964; Canguilhem, 1976; Monod, 1985; Maynard, 1987; Valencia, 1989; Jiménez y Sanmartí, 1997; García, 1998; Mayr, 1998, 2006; Capra, 2000; Wandersee, Fischer, y Moody, 2000; Valencia et al, 2001; Varela, 2002; Jiménez, 2003) se proponen los siguientes niveles en la hipótesis de progresión, para el caso de la estructura sustantiva del C.B.

El nivel inicial corresponde a generalizaciones fundamentalmente respecto la composición, la estructura, el funcionamiento y la clasificación de la vida, esto principalmente desde la perspectiva fisicoquímica. Dichas generalizaciones se reflejan en leyes y teorías expresadas en formulaciones matemáticas. Se hace énfasis en el abordaje del estudio de la vida, principalmente de manera analítica, ya sea mediante la observación o la experimentación, predominando el estudio de los elementos de una manera 


\begin{tabular}{|c|c|c|}
\hline Categoría & Nivel inicial & Nivel de referencia \\
\hline $\begin{array}{c}\text { Estructura } \\
\text { sustantiva del } \\
\text { conocimiento } \\
\text { biológico }\end{array}$ & $\begin{array}{l}\text { Enfoque fisicalista: } \\
\text { Énfasis en estudio analítico } \\
\text { de características de la vida. } \\
\text { Se produce observando y ex- } \\
\text { perimentando.Ausencia de un } \\
\text { estatus epistemológico propio. }\end{array}$ & $\begin{array}{l}\text { Enfoque sistémico y complejo: } \\
\text { Énfasis en estudio de las relacio- } \\
\text { nes que hacen posible lo vivo. Las } \\
\text { leyes no son preponderantes para } \\
\text { producir las teorías. Se produce: } \\
\text { experimentalmente, o mediante } \\
\text { enfoque histórico-narrativo. } \\
\text { Es epistemológicamente diferen- } \\
\text { ciado. }\end{array}$ \\
\hline $\begin{array}{l}\text { Producción del } \\
\text { conocimiento } \\
\text { biológico }\end{array}$ & $\begin{array}{l}\text { Visión empírico-positivista: } \\
\text { El conocimiento biológico es } \\
\text { producido por los investiga- } \\
\text { dores, directamente a partir } \\
\text { de la observación, la experi- } \\
\text { mentación, y/o la implement- } \\
\text { ación del método científico. }\end{array}$ & $\begin{array}{l}\text { Visión alternativa: } \\
\text { El conocimiento biológico es pro- } \\
\text { ducido mediante validación, como } \\
\text { un proceso histórico y cambiante, } \\
\text { utilizando metodologías diversas. } \\
\text { Para formular teorías no siempre } \\
\text { se requiere de experimentos, y son } \\
\text { fundamentales los conceptos. }\end{array}$ \\
\hline $\begin{array}{l}\text { Finalidades del } \\
\text { conocimiento } \\
\text { biológico }\end{array}$ & $\begin{array}{l}\text { Visión descriptiva: } \\
\text { La finalidad de la Biología es } \\
\text { producir conocimientos ge- } \\
\text { nerales o muy específicos (no } \\
\text { fundamentales) de la vida. }\end{array}$ & $\begin{array}{l}\text { Visión biopolítica: } \\
\text { La finalidad de la Biología es pro- } \\
\text { ducir: conocimientos, de lo vivo, } \\
\text { tanto fundamentales como aplica- } \\
\text { dos (de impacto en la sociedad); y } \\
\text { procedimientos para el desarrollo } \\
\text { de esta ciencia. Además promover } \\
\text { el desarrollo de actitudes favorables } \\
\text { para el conocimiento, conservación } \\
\text { y uso sostenible de los recursos } \\
\text { naturales. }\end{array}$ \\
\hline $\begin{array}{l}\text { Principales } \\
\text { contenidos del } \\
\text { conocimiento } \\
\text { biológico }\end{array}$ & $\begin{array}{l}\text { Enfoque aditivo- simplista: } \\
\text { Los principales contenidos } \\
\text { de la Biología son diversos } \\
\text { conceptos de la vida y la natu- } \\
\text { raleza y/o variados campos de } \\
\text { la Biología. }\end{array}$ & $\begin{array}{l}\text { Enfoque estructural- sistémico: } \\
\text { Los principales contenidos de la } \\
\text { Biología son: } \\
\text { - conceptos estructurantes de lo } \\
\text { vivo (especialmente desde la per- } \\
\text { spectiva sistémica). } \\
\text { - procedimientos y actitudes para } \\
\text { el conocimiento, conservación y uso } \\
\text { sostenible de lo vivo. } \\
\text { - aspectos históricos y episte- } \\
\text { mológicos de la Biología. }\end{array}$ \\
\hline
\end{tabular}


aislada, lo cual concuerda con una visión mecanicista de la Biología. En consecuencia, los principales referentes de estudio de este conocimiento son la Anatomía y la Taxonomía. En relación con su estatus epistemológico, no hay una identidad propia en el sentido que se le ve como una "ciencia auxiliar" la cual depende totalmente de ciencias como la Física y la Química. La vida es entendida desde la exactitud, desde una mirada fisicalista ${ }^{1}$. En el nivel de referencia se le asigna al C.B. un estatus epistemológico diferenciado, dado fundamentalmente por las características sistémicas propias de lo vivo en lo que tiene que ver con su objeto de estudio, e igualmente con especificidades propias en la manera como se produce; se considera que la Biología tiene una autonomía como ciencia, y a la vez con carácter interdisciplinario y aplicable (principalmente en las áreas de la salud, y lo ambiental).

Desde la perspectiva del nivel de referencia, dicho conocimiento no es exacto, sino por el contrario, relativo, diverso, multicausal, individual y particular, dadas las especificidades (prácticamente irrepetibles), cambios y variaciones (tanto individuales como generacionales) que ocurre en los seres vivos y de los fenómenos biológicos. Ello implica que no siempre hay generalizaciones a nivel de leyes, teorías y expresiones matemáticas, dándose en algunos casos las predicciones probabilísticas. En cuanto al énfasis de estudio, se refiere a la forma, los patrones de organización, las interrelaciones, la autoorganización, el automantenimiento, la retroalimentación, el conjunto y las emergencias.

En lo que atañe a la producción del C.B., teniendo presentes el marco de referencia teórico y los antecedentes de investigación (Blandino, 1964; Canguilhem, 1976; Monod, 1985; Serrano, 1985; Maynard, 1987; Benson, 1989; Jiménez y Sanmartí, 1997; García, 1998; Mayr, 1998, 2006; Capra, 2000; Wandersee, Fischer, y Moody, 2000; Herrera, Salcedo y Perafán, 2001; Varela, 2002; Jiménez, 2003) se propone la siguiente hipótesis de progresión.

El nivel inicial corresponde a una visión empírico-positivista, al considerar que el C.B. se produce a partir de la observación, ó experimentación de los seres vivos, ó de los fenómenos biológicos. Desde esta perspectiva, la fuente de conocimiento está en lo observado, es decir en lo vivo, tratándose entonces de hacer una transferencia directa del conocimiento desde el objeto a través de la percepción. Este nivel se corresponde con el nivel inicial de la hipótesis de progresión de la anterior categoría, al considerar que el C.B. es exacto y se puede alcanzar aplicando metodologías únicas, como es el caso del método científico. De otra parte, en este nivel se considera que una condición para la producción de C.B. es traducir dicho conocimiento en expresiones matemáticas para poder formular las leyes y las teorías.

En el nivel de referencia se tiene en cuenta que dadas las particularidades del objeto de estudio de la Biología, y por ende del C.B., no se pueden unificar metodologías para su

1 Este término es utilizado por Mayr (2006), y hace referencia a las Ciencias desde la perspectiva galiléica. 
producción. Por ejemplo, al existir especificidades y diferencias entre los organismos, las poblaciones y los fenómenos biológicos, resulta difícil garantizar uniformidad en las réplicas de determinado experimento, con lo cual, las condiciones experimentales presentan inconvenientes. Estas características, de igual forma implican enfoques probabilísticos en la producción del conocimiento. Así pues, existen diferentes metodologías para la producción del C.B., en el caso de los fenómenos de causación próxima (como los estudios en Fisiología, Citología, Embriología, etc), se acoge prioritariamente la experimentación (con las consecuentes limitaciones). En los estudios que involucran causaciones remotas (por ejemplo la evolución) principalmente se acude a los métodos comparativos, a la narración histórica. A diferencia del enfoque fisicalista galiléico de las Ciencias, en la Biología las leyes no tienen un papel preponderante en la producción de las teorías. Así, la mayoría de las teorías biológicas se basan en conceptos.

Respecto a las finalidades del Conocimiento Biológico, en concordancia con las fuentes bibliográficas consultadas (Blandino, 1964; Canguilhem, 1976; Jiménez y Sanmartí, 1997; Mayr, 1998; Capra, 2000; Wandersee, Fischer, y Moody, 2000; Valencia et al., 2001; Varela, 2002; Jiménez, 2003; Hall et al., 2003; Cañal, 2004), se propone, un nivel inicial que hace referencia al propósito que tiene el C.B. en el sentido exclusivo de describir y explicar las características de la vida, fundamentalmente desde la perspectiva de su composición, estructura y clasificación. Esto desde una perspectiva analítica y naturalista, es decir abordando el estudio de los elementos constituyentes de la vida de una forma inconexa, lo cual significa que no se tiene un referente del todo (como unidad). Desde esta perspectiva la finalidad del conocimiento biológico se refleja en la producción fundamentalmente de conceptos. Desde el punto de vista del nivel de referencia, la finalidad del C.B. no solamente se centra en producir los conceptos fundamentales relacionados con lo vivo, sino que además trasciende aportando elementos de aplicación en diversos campos como son la salud, lo ambiental, la agricultura, etc., que conlleva a tener un impacto en la sociedad, derivando en la producción de técnicas y tecnologías, tendientes a mejorar las condiciones de vida del hombre. Además, en lo que atañe a los conceptos básicos, se busca que estos no se limiten a la composición y estructura de los organismos y de los fenómenos biológicos, sino que tengan en cuenta la organización desde un enfoque sistémico. De igual manera, desde esta perspectiva integradora se pretende, además de producir conceptos, producir procedimientos propios de la Biología y actitudes y valores que promuevan el uso sostenible de los recursos.

En cuanto a los principales contenidos del C.B., la hipótesis de progresión fue elaborada a partir de la revisión bibliográfica (Blandino, 1964; Laborit, 1970; Canguilhem, 1976; Monod, 1985; Maynard, 1987; Jiménez y Sanmartí1997; Mayr, 1998; Capra, 2000; Wandersee, Fischer, y Moody, 2000; Varela, 2002; Jiménez, 2003), y de los antecedentes sobre estudios de la estructura del C.B. en profesores de Biología (Hashweh, 1987; Hauslein, Good, y Cummins, 
1992; Gess-Newsome y Lederman, 1993; Lederman, Gess-Newsome y, Latz,1994; Gess-Newsome y Lederman, 1993). En el nivel inicial se presenta una "visión atomizada" de conceptos biológicos y de campos de la Biología, lo cual implica considerar que los contenidos del C.B. no están organizados de forma coherente ni sistemática, sino que se conciben como un cúmulo de conceptos y de ramas de la Biología, a manera de listado simple.

En el nivel de referencia se hace énfasis en características fundamentales de lo vivo desde la perspectiva sistémica, lo cual conlleva a identificar conceptos como: la autoorganización, la autorregulación, la unidad como sistema, los patrones de organización, las propiedades emergentes, la autopoiesis, los programas genéticos y la herencia, la biodiversidad, y la evolución, entre otros. También son prioritarios los aspectos históricos y epistemológicos. Desde esta perspectiva, en contraste con el nivel inicial, se prioriza en las relaciones, más que en la la composición. Respecto a la clasificación de campos de la Biología, dada su amplitud, en este nivel se propone que sea diversa y organizada obedeciendo a diferentes criterios tales como: el tipo de causación: ramas como la Embriología, la Biología Celular, la Fisiología, etc. que corresponden a causas próximas (en la clasificación de Mayr, 2006, corresponde a la Biología funcional), o bien ramas como la evolución que está relacionada con causas remotas (Biología evolutiva, según la clasificación de Mayr, 2006); los niveles jerárquicos de organización (desde lo molecular hasta lo astrobiológico); las finalidades; la forma de producción del conocimiento biológico; y el objeto de estudio.

\section{Hipótesis de Progresión sobre los componentes del Conocimiento Didáctico del Contenido Biológico (CDCB).}

En la Tabla 2. se muestran la hipótesis de progresión de las respectivas categorías de investigación.

En el nivel inicial se plantea que el Conocimiento Profesional específico que requiere un profesor de Biología es saber el C.B. únicamente. En esta idea, basta con que el profesor domine académicamente el conocimiento de la Biología para que sea un buen profesor de Biología. Desde esta perspectiva, la organización y secuenciación del currículo obedece única y exclusivamente a la lógica de la Biología; es decir: se presenta un reduccionismo biológico. No se requiere así, de un conocimiento pedagógico y didáctico, asumiendo entonces que la forma de enseñar (restringida a explicar) obedece solamente a características personales del profesor.

El nivel de referencia corresponde a la necesidad de un CDCB, el cual se caracteriza por ser epistemológicamente diferenciado y por hacer posible la transformación e integración didáctica de los saberes y conocimientos provenientes de diferentes fuentes (científica, curricular, cotidiana, cultural, contextual). Dicho conocimiento está conformado por diversos componentes: contenidos que se enseñan en la clase de Biología, estrategias metodológicas y finalidades de la enseñanza de la Biología, conocimiento de las características de los alumnos (ideas, intereses, experiencias respecto a los contenidos biológicos que se enseñan), dificultades en la enseñanza y el aprendizaje de la Biología, y evaluación 


\section{Categoría}

Componentes del CDCB

Finalidades de la enseñanza de la

Biología

Enfoque

metodológico

de enseñanza-

aprendizaje de la

Biología

Conocimiento de los estudiantes a enseñar Biología.

Evaluación de los aprendizajes de la Biología.

Conocimiento Biológico Escolar
Reduccionismo Biológico: Para enseñar Biología basta con saber Biología.

\section{Perspectiva nominalista:}

El principal propósito de la enseñanza de la Biología es que los alumnos aprendan los contenidos (esencialmente conceptuales) de la Biología, entendidos como términos especializados.

Perspectiva transmisionista:

La enseñanza de la Biología consiste en la explicación de los contenidos estipulados en los libros de texto y en los programas curriculares.

Los alumnos aprenden Biología directamente al observar el objeto de aprendizaje o al recibir información.

Reduccionismo nominalista: En la clase lo importante es enseñar términos especializados de la Biología.

El estudiante como tábula rasa. Lo importante es transmitir contenidos a los estudiante, sin ser fundamental las diferencias entre sujetos.

Evaluación sumativa y de control: La evaluación de los aprendizajes de la Biología es de carácter sumativo y cuantitativo; sin autoevaluación; tiene modalidad de examen; busca control sobre los alumnos y que ellos reproduzcan la información enseñada.

Perspectiva simplificadora del conocimiento científico: El conocimiento de Biología que se enseña en la escuela corresponde a la repetición de términos especializados producidos por científicos y que aparece en el material curricular prescrito.

\section{Nivel de referencia}

Perspectiva integradora:

Para enseñar Biología, es necesario un CDCB, producido por la integración y transformación de conocimientos académicos (Biología, Pedagogía, Didáctica y otros saberes) y experienciales, que posibilite la transformación didáctica para producir conocimiento escolar biológico.

Perspectiva significativa-sistémica:

Los principales propósitos de la enseñanza de la Biología consisten en que los alumnos:

-Interrelacionen de manera integral los contenidos conceptuales, procedimentales y actitudinales fundamentales de la Biología.

-Encuentren aplicabilidad a la Biología en su vida cotidiana y en la conservación y aprovechamiento sostenible de los recursos naturales.

-Establezcan relaciones CTS.

Perspectiva constructivista: La enseñanza de la Biología consiste en la organización de objetivos y contenidos formativos, $y$ la implementación de actividades orientadas al aprendizaje significativo, al desarrollo metacognitivo y a la construcción de conocimiento escolar.Tiene en cuenta las características del alumno, del conocimiento biológico y del contexto.

Los alumnos aprenden Biología a partir de la reestructuración de sus concepciones como proceso metacognitivo e idiosincrásico.

Perspectiva integradora: En la clase de Biología se enseñan conceptos biológicos fundamentales (que explican el fenómeno de lo vivo en sus diferentes niveles de organización), procedimientos propios del estudio de lo vivo y actitudes frente al conocimiento, conservación y aprovechamiento sustentable de lo vivo.

Conocer las características de los estudiantes: condición "sine quanum" para la enseñanza. Para enseñar Biología en la escuela, es fundamental conocer las concepciones, experiencias e intereses de los estudiantes respecto a los contenidos que se enseñan.

Evaluación constructiva: La evaluación de los aprendizajes de la Biología es de carácter procesual, continuo, formativo y reflexivo; fundamentalmente cualitativo. Incluye autoevaluación, evaluación de profesor y coevaluación. Principalmente se utiliza como regulación, para la mejora del proceso de enseñanza-aprendizaje.

Perspectiva integradora-transformadora de diferentes tipos de conocimientos y saberes.

El conocimiento de Biología que se enseña en la escuela resulta de la transformación e integración didáctica de los conocimientos científicos, cotidianos, contextuales, $y$ otros.

Tabla 2. Hipótesis de Progresión acerca del Conocimiento Didáctico del Contenido Biológico (CDCB). 
de los aprendizajes de Biología. En este conocimiento confluyen, se transforman, e integran los diversos componentes que hacen posible la construcción del Conocimiento Profesional del profesor de Biología.

\section{Conclusiones}

Las investigaciones sobre el conocimiento del profesor de Biología, mayoritariamente se refieren a la estructura sustantiva del conocimiento biológico, especialmente en lo que atañe a la manera como los docentes estructuran conceptos biológicos, y a la comprensión que tienen de los mismos, siendo en la mayoría de los profesores deficientes.

Los resultados de investigación coinciden en que el conocimiento biológico del profesor es especialmente relevante en la enseñanza, en tanto incide en la selección y organización de contenidos, así como en sus estrategias de enseñanza.

Existen escasos estudios sobre el conocimiento didáctico del contenido biológico, haciéndose necesario construir conocimiento al respecto, a partir de la caracterización y análisis del pensamiento y las prácticas docentes de los profesores de Biología.

Aunque las hipótesis de progresión propuestas no involucran todos los componentes del conocimiento del profesor de Biología, se corresponden con los componentes más distintivos de dicho profesor, al hacer referencia a las características que identifican el conocimiento disciplinar específico y su enseñanza particular. En ese sentido, constituyen un referente teórico y metodológico para abordar investigaciones sobre el conocimiento del profesor de Biología.

En cada componente del conocimiento del profesor de Biología abordado, las hipótesis de progresión han sido formuladas tomando como soporte los hallazgos encontrados en la revisión de antecedentes de investigaciones sobre profesores de Biología, así como los planteamientos propios de teóricos de la enseñanza de las Ciencias y de la historia y epistemología de la Biología.

Dado que las hipótesis de progresión presentadas han sido formuladas estrictamente a partir de una revisión documental, no son suficientes para dar cuenta de la caracterización del conocimiento del profesor de Biología. Así, se hace necesario enriquecer dichas hipótesis a partir de investigaciones con docentes.

\section{Bibliografía}

Abell, S. (2007). Research on Science Teacher Knowlege. In: Abell, S., and Lederman, N. Handbook of Research on Science Education. New York, London: Routledge. pp. 1105-1149.

Abell, S. (2008). Twenty year latter: Does pedagagogica content knowledge remain a useful idea? International Journal of Science Education. 30 (10), 1405-1416

Aikenhead, G. (2007). Expanding the research agenda for scientific literacy. In Douglas, R. Promoting scientific literacy: science educations research in transactionUppsala Sweden: Uppsala University. pp. 64-71.

Amórtegui, E. (2011). Estudio de concepciones de futuros profesores de 
Biología acerca de las prácticas de campo. Tesis para optar al título de Magister en Educación. Documento inédito. Bogotá: Universidad Pedagógica Nacional.

Amórtegui, E. y Correa, M. (2009). Caracterización y análisis de las prácticas de campo en el ciclo de fundamentación del Proyecto Curricular de Licenciatura en Biología de la Universidad Pedagógica Nacional, en el marco de la construcción del Conocimiento Profesional del Profesor de Biología. Trabajo de grado para optar al título de Licenciada en Biología. Documento inédito. Bogotá: Universidad Pedagógica Nacional.

Barass, R. (1984). Some misconceptions and misunderstandings perpetuated by teachers and textbooks in biology. Journal of Biological Education, 13, 201-206.

Barnett J. y Hodson, D. (2001) Pedagogical Context Knowledge: Toward a Fuller Understanding of What Good Science Teachers Know. Science Education, 85 (4), 426-453.

Benson, G. (1989). Epistemology and science curriculum. Journal of Curriculum Studies, 21 (4), 329-344.

Bernal, C. (2007). Estudio de las concepciones sobre el Conocimiento Biológico en docentes en formación en el sector rural. Trabajo de grado para optar al título de Licenciada en

Biología. Documento inédito. Bogotá: Universidad Pedagógica Nacional.

Blandino, G. (1964). Problemas y teorías sobre la naturaleza de la vida. Una exposición crítica de la moderna biología teórica. Madrid: Razón y Fe S.A.

Canguilhem, G. (1976). El conocimiento de la vida. Barcelona: Anagrama.
Cañal, P. (2004). La enseñanza de la biología: ¿cuál es la situación actual y qué hacer para mejorarla?. Alambique, 41, 27-41.

Capra, F. (2000). La trama de la vida. Una perspectiva de los sistemas vivos. Quinta edición. Barcelona: Anagrama.

Carlsen, W. (1991). Effects of New Biology Teachers' Subject-Matter Knowledge on Curricular Planning. Science Education, 75 (6), 631-647.

Carlsen, W. (1993). Teacher Knowledge and Discourse Control: Quantitative Evidence From Novice Biology Teachers' Classrooms. Journal of Research in Science Teaching, 30 (5), 471-481.

Carlsen, W. (1999). Domains of Teacher Knowledge. In: Gess-Newsome, J.; and Lederman, N. (Eds.). Examining Pedagogical Content Knowledge. The Construct and its Implications for Science Education. Dordrecht, Boston, London: Kluwer Academic Publishers. pp. 133-144.

Castro, A. y Valbuena, E. (2007) ¿Qué biología enseñar y cómo hacerlo? Tecné Episteme y Didaxis. 22 (1), 126-145.

Castro, J.; Valbuena, E.; Gutiérrez, A.; Ruiz, D., Correa, M. y Morales, D. (2009). Construcción del conocimiento didáctico del contenido biológico en formación inicial de profesores. Enseñanza de las Ciencias, Número Extra VIII Congreso Internacional sobre Investigación en Didáctica de las Ciencias, Barcelona, pp. 3205-3208

http://ensciencias.uab.es/congreso09/ numeroextra/art-3205-3208.pdf.

Chona, G.; Castaño, C.; Arteta, J.; Leudo, M.; Valencia, S. Y Martínez, S. (1998). Aproximación a las creencias que orientan la práctica del profesor de 
biología. Tecné Episteme y Didaxis. 4, 93-104.

Cobern, W. and Loving, A. (2001). Defining "Science" in a Multicultural World: Implications for Science Education. Science Education 85: 50-67.

Douvdevany, O., Dreyfus, A, y Jungwirth, E. (1997). Diagnostic instrument for determining junior high-school science teachers' understanding of functional relationships within the "living cell". International Journal of Science Education, 1999, 503-606.

El-Hani, Ch. y Sepúlveda, C. (2006). Referenciais teóricos e subsídios metodológicos para a pesquisa sobre as relações entre educação científica e cultura. Em: Texeira, Greca, organizadoras. A Pesquisa em ensino de ciências no Brasil e suas Metodologias. Ijui: Editora UNIJUI. PP. 161-212.

Fenstermacher, G. (1994). The knower and the known: The nature of Knowledge in research on teaching. In: L. DarlingHammond (Ed.), Review of research in education Washington, DC: American Educational Research Association. Vol. 20, pp. 3-56.

García, E. (1998). Hacia una teoría alternativa sobre los contenidos escolares. Sevilla: Díada.

Gess-Newsome, J. (1999a). Pedagogical Content Knowledge: An introduction and orientation. In: Gess-Newsome, J.; and Lederman, N. (Eds.). Examining Pedagogical Content Knowledge. The Construct and its Implications for Science Education. Dordrecht, Boston, London: Kluwer Academic Publishers. pp. 3-17.

Gess-Newsome, J. (1999b). Secundary Teachers' Knowledge and beliefs about Subjet Matter and their Impact on Instruction. In: Gess-Newsome, J.; and Lederman, N. (Eds.). Examining Pedagogical Content Knowledge. The Construct and its Implications for Science Education. Dordrecht, Boston, London: Kluwer Academic Publishers. pp. 51-94.

Gess-Newsome, J. y Lederman, N. (1993) Preservice Biology Teachers' Knowledge Structures as a Function of Professional Teacher Education: A Year-Long Assessment. Science Education, 77 (1), 25-45.

Gess-Newsome, J. y Lederman, N. (1995). Biology Teachers' Perceptions of Subject Matter Structure and its Relationship to Classroom Practice. Journal of Research in Science Teaching, 32 (3), 301-325

Gess-Newsome, J. y Lederman, N. (Eds.) (1999). Examining Pedagogical Content Knowledge. The Construct and its Implications for Science Education. Dordrecht, Boston, London: Kluwer Academic Publishers.

Grossman, P. (1990). The Making of a Teacher. Theacher Knowledge and Teacher Education. New York: Teachers College, Columbia University.

Gutiérrez, A. (2007). Indagación y análisis de referentes sobre el Conocimiento Disciplinar y su relación con el Conocimiento Profesional en docentes en formación inicial del proyecto curricular de Licenciatura en Biología de la Universidad Pedagógica Nacional. Trabajo de grado para optar al título de Licenciada en Biología. Documento inédito. Bogotá: Universidad Pedagógica Nacional.

Hall A.; Reiss, J.; Rewell C.; y Scott A. (2003). Biología avanzada Salters-Nuffield: un nuevo curso de biología para la etapa de 16-18 años, Alambique. 
Hashweh, M. (1987). Effects of subjectmatter knowledge in the teaching of biology and physics. Teaching \& Teacher Education, 3 (2), 109-120.

Hauslein, P., Good, R. y Cummins, C. (1992). Biology Content Cognitive Structure: From Science Student to Science Teacher. Journal of Research in Science Teaching, 29 (9), 939-964.

Herrera, L., Salcedo, L, y Perafán, A. (2001). Acciones y Creencias. Análisis e interpretación de creencias de docentes en Biología y Ciencias Naturales. Bogotá: Universidad Pedagógica Nacional.

Hoz, R. Tomer, Y. y Tamir, P. (1990). The relations between disciplinary and pedagogical knowledge and the length of teaching experience of biology and geography teachers. Journal of Research in science teaching, 27 (10), 973-985.

Jiménez, M.P. (2003). La enseñanza y el aprendizaje de la biología. En: Jiménez, M.P. (Coordinadora) Enseñar ciencias. Barcelona: Graó. pp. 119146.

Jiménez, M.P. y Sanmartí, N. (1997). ¿Qué ciencia enseñar?: objetivos y contenidos en la Educación Secundaria, En: Del Carmen, L (Coordinador) La enseñanza y el aprendizaje de las ciencias de la naturaleza en la educación secundaria, Barcelona: Horsori editorial. pp. 17-46.

Laborit, H. (1970). Biología y Estructura. Caracas: Tiempo Nuevo.

Magnusson, S.; Krajcik, J.; y Borko, H. (1999). Nature, Sources, and Development of Pedagogical Content Knowledge for Science Teaching. In: Gess-Newsome, J.; and Lederman, N. (Eds.). Examining Pedagogical Content Knowledge. The Construct and its Implications for Science Education.
Dordrecht, Boston, London: Kluwer Academic Publishers. pp. 95-132.

Marcelo, C. (1987). El pensamiento del profesor. Barcelona: Ceac.

Marcelo, C. (1999). Cómo conocen los profesores la materia que enseñan. Algunas contribuciones de la investigación sobre conocimiento didáctico del contenido. En: Montero, L.; y Vez, J. (Eds.). Las didácticas específicas en la formación del profesorado. Santiago de Compostela: Tórculo. pp. 151-185.

Maynard-Smith, J. (1987). Los problemas de la biología. Madrid: Cátedra.

Mayr, E. (1998). Así es la biología. Barcelona: Debate.

Mayr, E. (2006). Por qué es única la biología. Buenos Aires: Katz.

Monod, J. (1985). El azar y la necesidad. Ensayo sobre la filosofía natural de la biología moderna. Barcelona: Tusquets.

Morales, D. (2010). La visita a un museo de historia natural. Un estudio de caso desde la perspectiva del conocimiento didáctico del contenido. Trabajo de grado para optar al título de Licenciada en Biología. Documento inédito. Bogotá: Universidad Pedagógica Nacional.

Morales, D. y Valbuena, É. (2009). Referentes del Conocimiento Biológico en futuros profesores de Biología. En: Memorias del Primer Congreso Nacional de Investigación en Educación en Ciencia y Tecnologías. Educyt, Cali, 22-24de Junio.

Puentes, M. (2008). Los trabajos prácticos en la enseñanza de la Biología. Trabajo de grado para optar al título de Especialista en Enseñanza de la Biología. Documento inédito. Bogotá: Universidad Pedagógica Nacional. 
Morine-Dershimer, G. y Kent, T. (1999). The Complex Nature and Sources of Teachers' Pedagogical Content Knowledge. In: Gess-Newsome, J.; And Lederman, N. (Eds.). Examining Pedagogical Content Knowledge. The Construct and its Implications for Science Education. Dordrecht, Boston, London: Kluwer Academic Publishers. pp. 21-50.

Lederman, N.; Gess-Newsome, J. y Latz, M. (1994). The Nature and Development of Preservice Science Teachers' Conceptions of Subject Matter and Pedagogy. Journal of Research in Science Teaching, 31 (2), 129-146.

Lee O., and Luykx, A. (2007). Science Education and Student Diversity: Race/ Ethnicity, Language, Culture, and Socioeconomic Status. Abell, S., and Lederman, N. Handbook of Research on Science Education. New York, London: Routledge. pp. 171-198.

Oliver, J. (2007). Rural Science Education. In: Abell, S., and Lederman, N. Handbook of Research on Science EducationNew York, London: Routledge. pp. 345-371.

Park S. and Oliver, S. (2008). Revisiting the Conceptualisation of Pedagogical Content Knowledge (PCK): PCK as Conceptual Tool to Understand Teachers as Profesionals. Research on Science Education. 38: 261-284.

Porlán, R. y Rivero, A. (1998). El conocimiento de los profesores: una propuesta formativa en el área de ciencias. Sevilla: Díada.

Puentes, M. (2008). Los trabajos prácticos en la enseñanza de la Biología. Trabajo de grado para optar al título de Especialista en Enseñanza de la Biología. Documento inédito. Bogotá: Universidad Pedagógica Nacional.
Sánchez, P. (2007). Formulación de proposiciones para el estudio de las concepciones sobre el Conocimiento Biológico en el marco del Conocimiento Profesional del Profesor de Biología. Trabajo de grado para optar al título de Licenciada en Biología. Documento inédito. Bogotá: Universidad Pedagógica Nacional.

Sanders, M. (1993). Erroneous ideas about respiration. The teacher factor. Journal of Research in Science Teaching, 30, 919-934.

Shulman, L. (1986a). Those who understand: Knowledge growth in teaching. Educational Researcher, 15 (2), 4-14.

Shulman, L. (1986b). Paradigms and research programs in the study of teaching: A contemporary perspective. In: Wittrock, M. (Ed.) Handbook of Research on Teaching. New York: Macmillam. pp. 3-36.

Tardif, M. (2004). Los saberes del docente y su desarrollo profesional. Traducción de Pablo Manzano. Madrid: Narcea.

Valencia, S. (1989). La Biología: ¿ciencia de la vida o ciencia de lo vivo?. Análisis de condiciones epistemológicas que hicieron posible pensar los determinantes del fenómeno vivo como el objeto de lo vivo. Bogotá: Imprenta Universidad Pedagógica Nacional.

Valencia, S.; Méndez, O.; Garzón, P.; y Jiménez-Gómez, G. (2001). De la contemplación a la comprensión de los seres vivos. Campo Abierto, 20, 31-46.

Valbuena, E. (2007). El Conocimiento Didáctico del Contenido Biológico. Estudio de las concepciones disciplinares y didácticas de futuros docentes de la Universidad Pedagógica Nacional (Colombia). Tesis doctoral. Univer- 
sidad Complutense de Madrid (ISBN 978-84-669-3101-4).

Valbuena, E (2009 a). Línea de investigación Conocimiento Profesional del Profesor de Ciencias. Bio-grafía. Escritos sobre Biología y su enseñanza. 2 (2), 2-15 (ISSN 2027-1034). http:// www.pedagogica.edu.co/revistas/ ojs/index.php/bio-grafia/article/ view/161.

Valbuena, E. (2009 b). Referentes de futuros profesores sobre el conocimiento biológico y el conocimiento didáctico del contenido biológico. Enseñanza de las Ciencias, Número Extra VIII Congreso Internacional sobre Investigación en Didáctica de las Ciencias, Barcelona, pp. 3091-3094

http://ensciencias.uab.es/congreso09/ numeroextra/art-3091-3094.pdf.

Valbuena, E., y Castro, A. (2007). La Importancia de los aspectos epistemológicos en la formación inicial de profesores de biología. Tecné Episteme y Didaxis. Número Extra. 3er Congreso Internacional de Formación de Profesores de Ciencias (ISSN 0121-3814).

Valbuena, E.; Castro, J.; y Sierra, C. (2006). El conocimiento biológico desde la perspectiva del conocimiento profesional del profesor de biología. Memorias $2^{\circ}$ Congreso Internacional de Enseñanza de la Biología. Neuquén, Argentina.

Valbuena, E; Correa, M; Amórtegui , E; Bernal, S. (2010a). Estado del arte del campo de conocimiento de la enseñanza de la Biología. Informe de investigación. Documento inédito. Bogotá: Universidad Pedagógica Nacional.
Valbuena, É., Gutiérrez, A., Correa, M., y Amórtegui, Elías. (2010b). Procesos formativos que favorecen la construcción del conocimiento profesional del profesor en futuros docentes de Biología. En: Revista Colombiana de Educación. No 56. Pp 156-179. ISSN: 0120-3916.

Varela, F. (2002). El fenómeno de la vida. $2^{a}$ edición. Caracas, Montevideo, Santiago de Chile: Dolmen.

Veal, W. y Kubasko, D. (2003). Biology and Geology Teachers' Domain-Specific Pedagogical Content Knowledge of Evolution. Journal of Curriculum and Supervision, 18 (4), 334-352.

Wandersee, J.; Fischer, K.; y Moody, D. (2000). Mapping Biology Knowledge. Dordrecht, Boston, London: Kluwer Academic Publishers. 